\title{
Quand la pharmacie clinique rencontre la pharmacologie clinique
}

\author{
Barbara Lardi-Studler \\ Dr sc. nat., pharmacienne, auteure indépendante
}

Les exigences croissantes dues aux thérapies toujours plus complexes, à l'augmentation du nombre de patients chroniques, à la polymorbidité ou encore aux difficultés d'approvisionnement représentent de gros défis pour les spécialistes du médicament. La pharmacie clinique développe et promeut l'utilisation sûre, rationnelle et économique des médicaments (ESCP); ces objectifs généraux sont également poursuivis par les pharmacologues cliniques. Le plus important est de stimuler le dialogue entre la pharmacie clinique et la pharmacologie clinique afin d'exploiter des synergies, éviter les licenciements et simplifier les procédures. Seulement de cette façon le meilleur approvisionnement en médicaments peut être assuré.

\section{Les pharmaciens cliniques et les pharma- cologues cliniques à l'hôpital}

(Résumé des exposés du Dr phil. Markus Lampert, du Prof. Dr med., Dr pharm. Stephan Krähenbühl, du Prof. Dr Chantal Csajka, du Prof. Dr med. Thierry Buclin et de Delia Bornand, pharmacienne diplômée FPH en pharmacie hospitalière)

Les termes de pharmacie clinique et de pharmacologie clinique ne créent pas seulement des confusions chez les patients - ils sont aussi source de malentendus pour les professionnels de la santé. La pharmacodynamie et la pharmacocinétique font certes partie intégrale des sciences pharmaceutiques, mais ce sont essentiellement les pharmacologues qui en ont la charge dans les grands hôpitaux. Il est toutefois important de définir précisément ce que recouvre ces deux domaines d'activités, différents par certains côtés mais similaires par d'autres, pour pouvoir utiliser au mieux les ressources limitées en personnel.

Environ $10 \%$ des patients subissent une complication médicamenteuse (Adverse Drug Events, ADE). Le pourcentage d'erreurs de prescriptions de médicaments est équivalent. Il y a donc de toute façon bien assez à faire pour ces deux groupes d'experts du médicament.

Même si la situation varie considérablement selon la taille de l'hôpital, le pharmacologue clinicien est plus directement en contact avec le patient alors que le pharmacien clinicien se souciera davantage des aspects liés à la sécurité des traitements médicamenteux. Aujourd'hui, au sein des grandes structures hospitalières universitaires, les pharmaciens et les pharmacologues cliniciens travaillent de manière complémentaire. Mais dans les établissements hospitaliers plus petits, les pharmaciens cliniciens doivent souvent se charger des tâches des pharmacologues en l'absence de département de pharmacologie clinique.

Le pharmacien clinicien connaît le processus de médication dans son intégralité. Il sert de lien entre les autres intervenants du processus. Exerçant une profession médicale universitaire et titulaire du certificat de formation complémentaire FPH en pharmacie clinique, il dispose des compétences nécessaires pour comprendre les risques liés aux médicaments aussi bien au niveau individuel (risques liés au patient, interactions, effets indésirables, etc.) que systémique (processus au sein de l'hôpital).

Par des interventions ciblées, le pharmacien clinicien peut contribuer à améliorer la sécurité du processus de médication et des traitements médicamenteux. Les expériences montrent que, selon la clinique, les recommandations des pharmaciens cliniciens participant à la visite des patients sont suivies et appliquées jusqu'à $85 \%$ des cas. Certains traitements médicamenteux requièrent une compréhension clinique approfondie, et c'est à ce stade qu'intervient le pharmacologue clinicien.

Outre les connaissances de base, cette formation postgrade a comme principaux objectifs l'évaluation des risques et bénéfices individuels du patient, la collaboration à des essais sur les médicaments, la préconisation de traitements individualisés et l'évaluation de la sécurité et de l'économicité des traitements médicamenteux.

Il est souhaitable de recourir aux conseils du pharmacologue clinicien pour résoudre les questions complexes (par ex. en cas d'insuffisances organiques), gérer les interactions et les effets indésirables chez les patients polymorbides ou pour interpréter les monitorings thérapeutiques. 
Les observations pertinentes qui ont été faites doivent absolument être publiées. Au CHUV par exemple, un site internet a été créé à partir de ces interventions et permet de calculer les adaptations de dose d'anti-infectieux chez des patients dialysés (www.crrt.ch).

Les pharmacologues cliniciens sont aussi des médecins exerçant une activité académique (enseignement et recherche), raison pour laquelle ils peuvent et doivent pour le moment exercer essentiellement dans des hôpitaux universitaires.

Toutefois, il arrive souvent que les patients ne séjournent pas assez longtemps à l'hôpital pour pouvoir discuter de leur médication avec un pharmacologue clinicien. Un constat qui laisse entrevoir de grosses opportunités pour les pharmaciens cliniciens, pas seulement à l'hôpital, mais aussi à l'officine!

\section{Le pharmacien clinique en officine}

(Exposé du Prof. Dr Olivier Bugnon)

Le pharmacien clinicien peut fournir une série de prestations très utiles au système de santé, comme:

- Analyses de médication;

- Consultations d'adhésion;

- Suivis de patients atteints de maladies chroniques, VIH compris;

- Traitement individualisé adapté spécialement aux groupes vulnérables;

- Gestion des risques;

- Echange à propos de situations problématiques et collaboration avec les médecins (cercles de qualité).

Le suivi pharmaceutique des patients après une hospitalisation constitue un grand pan de la pharmacie clinique ambulatoire. Souvent, une à deux semaines s'écoulent jusqu'à la prochaine consultation chez le médecin de famille. Durant ce laps de temps, le patient est souvent livré à lui-même, il a encore à la maison ses

Formation postgrade FMH en pharmacologie et toxicologie cliniques

Prérequis: bases de la pharmacologie acquises lors du Bachelor et du Master en médecine.

Durée: au moins 6 ans de formation postgrade ( 3 ans de formation en médecine interne, pédiatrie ou anesthésiologie et 3 ans de formation spécifique). La majeure partie des candidats suivent 5 ans de formation postgrade en médecine interne avant de poursuivre avec la formation postgrade en pharmacologie clinique. C'est primordial car la plupart des questions concerne la médecine interne et les pharmacologues cliniciens peuvent intervenir directement dans le processus de médication.

Nombre de candidats: 4 à 6 candidats formés par année.

Plus d'informations auprès de la SSPTC:

www.clinpharm.ch/Pharmaco/Formation anciens médicaments et n'est pas vraiment au clair avec les nouveaux qu'il doit désormais prendre. Ainsi, la Pharmacie de la PMU organise des visites au domicile des patients après la sortie de l'hôpital. Dans un premier temps, l'armoire à pharmacie familiale est triée, puis le traitement est abordé et expliqué au patient. Les expériences faites ont été très positives et les médecins généralistes ont beaucoup apprécié cette prestation.

A Lausanne, médecins, infirmières et pharmaciens se concertent pour optimiser leur collaboration après la sortie de l'hôpital.

\section{Aspects économiques}

(Exposé du Prof. Dr Christoph Meier)

Les aspects économiques deviennent de plus en plus importants. Si on part d'une incidence de $10 \%$ d'ADE et que l'on compte 5000 CHF de coûts supplémentaires par ADE, cela donne 19000 CHF par lit et par année. Pour un hôpital de la taille de celui de l'Université de Bâle, cela représente 12,5 millions de francs de coûts supplémentaires. Une somme considérable qui justifierait largement l'intervention systématique de pharmaciens et de pharmacologues cliniciens.

Cependant, les économies effectives réalisées par les pharmaciens cliniciens sont difficiles à montrer. Toutefois, ils permettent clairement de modifier certains objectifs des services où ils interviennent et ont un «effet de formation» sur le personnel soignant et le corps médical. Ce qui se reflète ensuite par une amélioration des traitements médicamenteux et un effet démultiplicateur au niveau de l'hôpital. En officine aussi, les pharmaciens bénéficiant d'une formation en pharmacie clinique sont des «multiplicateurs» importants.

\section{Conclusions}

Ces deux professions s'appuient sur la pharmacologie. A partir de ces connaissances de base, les deux spécialistes exercent chacune à leur manière. En particulier l'évaluation et la garantie de la sécurité des patients en général (évaluation de la posologie, EI, interactions, prescriptions multiples) et sur le plan individuel (par ex. pour les groupes vulnérables tels que les patients gériatriques, les femmes enceintes et les enfants). Ces activités sont aussi possibles pour les pharmaciens cliniciens qui exercent en officine.

A côté de ces grands traits communs, chaque spécialiste à des domaines plus clairement attribués. Ainsi les pharmacologues cliniciens sont des médecins qui, lorsqu'ils prescrivent un traitement médicamenteux, 


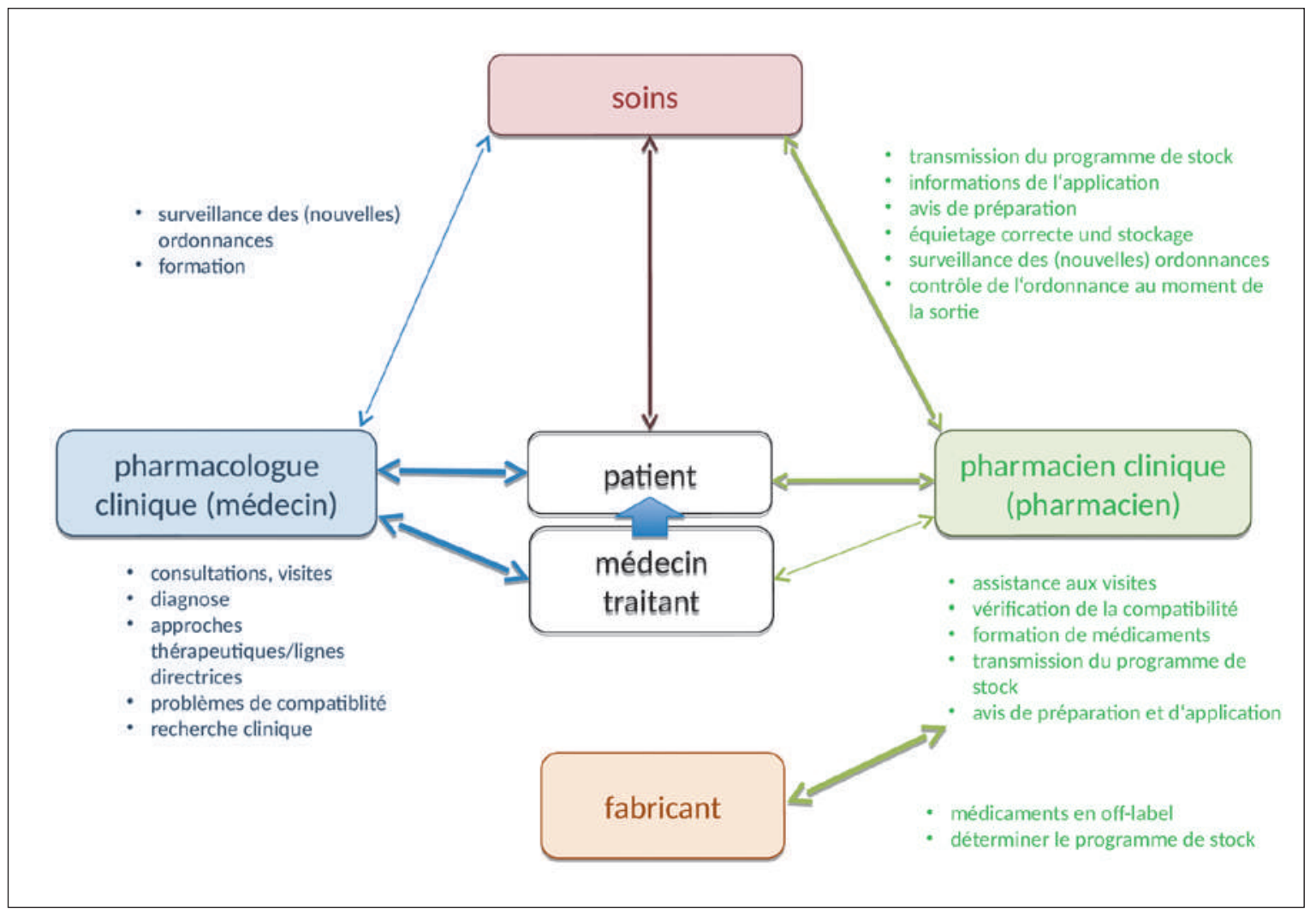

Figure 1: Le meilleur bénéfice pour le patient est provoqué par une bonne collaboration interdisciplinaire. Montrée est une sélection de ces interactions interdisciplinaires possibles entre les deux groupes professionnels pour optimiser la thérapie.

associent leurs connaissances pharmacologiques à tout le contexte clinique du patient, en particulier avec les diagnostics et les investigations effectuées.

Les pharmaciens cliniciens sont des pharmaciens qui se focalisent non seulement sur la pharmacologie mais aussi sur les aspects quotidiens et purement pratiques des traitements médicamenteux (fabrication, préparation du médicament, forme correcte). Ils mettent plus l'accent sur l'efficacité et la sécurité lors de l'utilisation du médicament et sur la promotion de l'adhésion au traitement.

A l'étranger, ce sont les pharmaciens cliniciens qui, dans de nombreux hôpitaux, prescrivent et surveillent les traitements médicamenteux. En Suisse, à l'heure actuelle, on tient compte des avis experts des deux

Certificat de formation complémentaire FPH en pharmacie clinique

Prérequis: bases de la pharmacologie acquises lors du Bachelor et du Master en pharmacie.

Durée: minimum 1 an après le Master pour un emploi à 100\%; formation toutefois essentiellement suivie à temps partiel spécialistes. Ce travail en commun est un énorme avantage pour notre système de santé. Les deux partenaires ont besoin l'un de l'autre et se complètent, pour le plus grand bénéfice du patient (fig. 1).

Il est donc souhaitable que le plus grand nombre possible de jeunes confrères et consœurs puissent suivre la formation postgrade d'une de ces deux professions. La mise sur pied des modules communs de formation de base et postgrade permettrait de promouvoir la communication interdisciplinaire et ainsi mieux comprendre et mieux utiliser les différentes manières de penser. Bien que nous soyons encore au stade des négociations pour savoir ce que ces deux professions doivent faire ou abandonner, les échanges et la collaboration devraient davantage être développées. L'ASSP voit comme une occasion nécessaire de rapprocher les deux professions en consensus avec l'ASSM et d'utiliser les synergies. Comme académie elle a la justification technique et scientifique et est ouverte aux deux professions. Une thérapie médicamenteuse supervisée par les deux professions est d'un grand bénéfice pour les patients et parle pour un bon résultat pharmaco-économique.

Version abrégée de la secousse préliminaire au pharmaJournal 8/15 (avec l'autorisation de pharmaSuisse). 